\title{
Calidad de vida en personas mayores en Chile
}

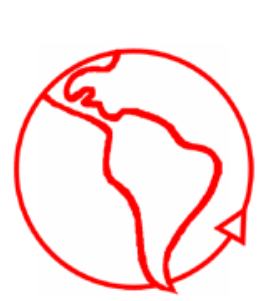

\author{
Quality of life in older people in Chile
}

\section{Paulina Osorio Parraguez}

Universidad de Chile, Facultad de Ciencias Sociales

María José Torrejón

Universidad de Chile, Facultad de Ciencias Sociales

\section{María Sol Anigstein}

Universidad de Chile, Facultad de Ciencias Sociales

\begin{abstract}
Resumen
La investigación cuyos resultados aquí se presentan, dice relación con la calidad de vida de vida de las personas mayores en Santiago de Chile, desde una perspectiva subjetiva, vale decir, desde el punto de vista de las propias personas de edad. La investigación plantea la hipótesis que existen otros factores gravitantes en la calidad de vida del adulto mayor que son tan importantes como los biomédicos, tales como: grados de autonomía, interacciones y redes sociales, condiciones económicas, vivienda y entorno urbano, actividades recreativas y de ocio, relación con cuidadores y capacidad de tomar decisiones en esta etapa de la vida, e incluso, la misma dimensión biográfica influiría en la percepción de calidad de vida. Estos hacen referencia a aspectos socioculturales particulares no sólo de un grupo etáreo, sino también, de una cultura. El desarrollo de la investigación ha contemplado el trabajo con dos técnicas de producción de datos cualitativos: grupos focales y entrevistas en profundidad. Para esta presentación se describirá y darán a conocer los resultados obtenidos a través de la realización de esta investigación cualitativa.
\end{abstract}

\begin{abstract}
The research, which results are presented here, is related to a subjective perspective of the quality of life of older people living in Santiago of Chile, ie the point of view of older people was studied. The research raises the hypothesis that other factors on older adults' quality of life are as important as biomedical, such as: autonomy degrees, interactions and social networks, economic conditions, housing and urban environment, recreational and leisure activities, relationship with caregivers, ability to make decisions at this stage of life, and even biographical dimension would influence in the perception of their quality of life. These factors refer to particular socio-cultural aspects not only of an age group, but also of a culture. The development of the study has considered working with two production techniques of qualitative data: focus groups and in-depth interviews. This presentation will describe the results obtained through the implementation of this qualitative research.
\end{abstract}

Palabras Clave: Personas mayores, calidad de vida, vejez, Chile, investigación cualitativa

Keywords: Older people, quality of life, aging, Chile, qualitative research 


\section{Introducción}

\section{a) Aproximaciones al concepto de calidad de vida}

La idea de calidad de vida es ampliamente utilizada en el ámbito científico, en los enunciados de la política pública y en la vida cotidiana en general. Dicha popularidad ha significado una abundante literatura al respecto y por lo mismo una gran cantidad de maneras de definirla (Dijkers 2007).

La popularización del concepto se vincula a transformaciones operadas durante el siglo XX que trasladan la atención desde temas relacionados con el ganarle a la muerte, como el hambre y la pobreza, a temas relacionados al cómo vivir la vida, al bienestar durante la vida y más aún, durante toda la vida. Si bien el concepto aparece en escena en los años sesenta (Hernández, 2004; Hidalgo, 2008), es en los setenta que los estudios sobre calidad de vida comienzan a cobrar importancia en el ámbito académico ${ }^{1}$. En la actualidad, a pesar de la prolífera producción de artículos e investigaciones sobre el tema desde diversas disciplinas, no ha sido posible establecer un consenso en relación a qué es y cómo se mide la calidad de vida (Aranibar 2004).

Aranibar (op. cit) considera que al utilizar el concepto de calidad de vida en el estudio de la vejez y el envejecimiento, se debe tener en cuenta tres premisas claves, que enmarquen la investigación. En primer lugar, la especificidad del concepto según su contexto de referencia, en este caso la vejez, que presenta diferencias con los otros grupos de edad en cuanto a aspectos y factores que configuran e intervienen la idea de calidad de vida. En segundo lugar, la multidimensionalidad de la calidad de vida, lo que significa que es preciso tomar en consideración tanto factores personales, como socioambientales o externos, factores que varían de acuerdo a la edad de las personas. Finalmente, el concepto de calidad de vida en la vejez, al igual que en todas las edades, debe considerar tanto aspectos subjetivos como objetivos, es decir, tanto las apreciaciones, valoraciones y evaluaciones sobre las distintas dimensiones de la calidad de vida, como los aspectos objetivos de las mismas. En esta misma línea, George (2006) afirma que hay dos maneras de aproximarse al concepto de calidad de vida, una que incluye la percepción subjetiva y las características objetivas y otra que se centra sólo en lo subjetivo. Pedrero (en Aranibar 2004), considera que observar la calidad de vida como algo puramente subjetivo puede llevar suponerla en alguien que reportara ser completamente feliz viviendo en la extrema pobreza, por lo que propone que para hablar de calidad de vida desde el punto de vista subjetivo sería necesario primero que las personas tuvieran cubiertas las necesidades mínimas, como alimentación, vivienda, servicios básicos, entre otros.

No obstante lo aquí mencionado, la Organización Mundial de la Salud (OMS) define calidad de vida como: "la percepción de cada individuo de su posición en la vida, en el contexto de la cultura y sistema de valores en el cual vive y en relación a las metas, expectativas, estándares y preocupaciones que tiene" (Schwartzmann 2003: 3) es decir, se centra en los aspectos subjetivos de la calidad de vida. Si bien consideramos que se trata de una definición bastante operativa y completa, pues considera el contexto sociocultural y cotidiano de los sujetos, creemos que al observar la calidad de vida, no se puede perder de vista la vida de ellos, vale decir, su lugar en el mundo en su momento biográfico. La trayectoria biográfica de cada persona constituye los lentes con los que observan y significan sus vidas y la calidad de éstas.

\footnotetext{
${ }^{1}$ Lo cual puede ser graficado con el aumento en la frecuencia de artículos referidos al tema específicamente en la literatura biomédica, psicológica y de ciencias sociales en general. A modo de ejemplo, en la base de datos Pubmed, los artículos que incluyen la frase "quality of life" en el título o abstract ascienden a un 0,002\% en 1966, pasando a ser un $1,36 \%$ en el año 2005 , sumando en total entre esos años 76.698 artículos que abordan el tema de la calidad de vida (Moons y otros 2006).
} 


\section{b) Calidad de Vida y Vejez}

El envejecimiento en Latinoamérica es un gran reto, si pensamos que la proporción de personas mayores de 60 años se triplicará entre los años 2000 y 2050, y uno de cada cuatro latinoamericanos será adulto mayor. El caso de Chile no es muy distinto, ya que se estima que en el año 2050 Chile tendrá una población adulta mayor superior al 28\%y que junto al incremento de la esperanza de vida y la disminución de los menores de 15 años, significará un envejecimiento poblacional de dimensiones considerables (CELADE 2006). Por ello, los mecanismos de inclusión y la consecuente integración se vuelven cruciales, cuando se piensa en la calidad de vida de las personas adultas mayores.

La calidad de vida ha sido medida e investigada en diferentes grupos de edad privilegiando los estudios en personas jóvenes. En el caso de las personas adultas mayores, uno de los grandes problemas que se identifica es lo inadecuado de algunos instrumentos de medición, pues no han sido diseñados necesariamente para evaluar la realidad y calidad de vida en este grupo etáreo. Puts y otros (2007) sostienen que aquello que es indicado y pudiera ser importante para la calidad de vida varía con la edad, siendo por ejemplo, en los jóvenes el encontrar trabajo y los asuntos financieros, y en los adultos mayores la salud y la movilidad.

Así, el abordaje de la calidad de vida en la vejez debe partir de la base de que si bien se trata de personas con una larga trayectoria de vida, es decir que han transitado por varias edades, y que por ello hay elementos y aspectos que intervienen en la calidad de vida comunes con otros grupos etáreos, existen especificidades en los adultos mayores que intervendrán en la calidad de vida de éstos, las cuales son necesarias de ser reveladas². Por lo que vemos, el envejecer, lejos de ser un proceso únicamente individual, es un proceso colectivo con implicancias y condicionado por el entorno social en el que tiene lugar, lo que significa que el tiempo individual se halla integrado al tiempo social, estando cada vivencia de las personas significada por el paso del tiempo y por el momento en que fueron experimentadas (Osorio 2001).

Los estudios de calidad de vida en la vejez están considerando tanto aspectos sociales como psicológicos. Siguiendo a Encarni Pedrero, esto se explica en el hecho de que la calidad de vida en la vejez está fuertemente ligada a factores sociales, económicos, culturales y psicológicos, siendo fundamental el sentido de coherencia, elemento que no se relaciona con las enfermedades o habilidades de la persona, sino más bien, con la capacidad de resistir ante las eventualidades que surgen y afrontar cambios duros, como por ejemplo, enfermedades nuevas (Pedrero 2001). Sin duda considerar el sentido de coherencia dentro del análisis de la calidad de vida adquiere mayor importancia cuando se refiere a la calidad de vida en la vejez, más que en los otros grupos de edad. Por lo tanto, la complejidad del concepto se vuelve mayor si lo cruzamos con esta realidad étarea. Julia Moreno (2004), percibe como fundamental dentro de la calidad de vida para los mayores la integración social, siendo posible generarla a través de actividades que produzcan en ellos placer y desarrollo tanto intelectual como social. Un punto importante que la autora destaca en relación a ello, es la importancia de las tecnologías, y su papel en el ocio, información y participación de los mayores.

Higgs y otros (2003) consideran que la sociedad cambia y con ella se modifican también las formas de vida, siendo más determinantes las variables sociales en la medición de la calidad de vida. Esto, al estudiar la calidad de vida en la vejez, significa que temas como el

\footnotetext{
${ }^{2}$ A modo de ejemplo podemos mencionar los resultados de un estudio realizado en Medellín, Colombia, donde se investigaron los componentes de la calidad de vida y de la condición de salud de la población adulta mayor. En él se concluyó que el $97 \%$ de la calidad de vida del adulto mayor de la ciudad es explicada por los componentes: autonomía, seguridad social-economía y redes de apoyo social (Cardona y otros 2002).
} 
ocio, que en otras generaciones era poco considerado, en la sociedad contemporánea ha adquirido una relevancia sin precedentes.

Actualmente, por ejemplo, el envejecimiento europeo se caracteriza por un retiro temprano del mercado laboral seguido de un largo período de buena salud posterior al retiro, al mismo tiempo, estas personas que inician su retiro, son más similares al resto de la población en términos de expectativas y motivaciones. Factores como estos llevan a pensar que la calidad de vida adquiere un significado particular en cada cohorte o generación (Walker 2005:28).

En relación a los indicadores de calidad de vida, un estudio bibliográfico realizado por Susana Sanduvette (2004), concluye que éstos se dividen en seis áreas: las opciones para tomar decisiones con respecto a su vida, contar con apoyo formal e informal y estar integrado a la sociedad, tener un estilo de vida de acuerdo con sus costumbres viviendo en un lugar de agrado y siendo independiente, gozar de la mayor salud posible y saber qué hacer en caso de emergencia, ejercen sus derechos y deberes sin recibir maltrato con servicios públicos adecuados y con la posibilidad de recibir ayuda legal, y, finalmente, la satisfacción medida en base a si las personas alcanzan sus objetivos que se marcan y están satisfechas con sus vidas y los servicios que reciben.

En el año 2007, la Pontificia Universidad Católica de Chile realizó la encuesta de calidad de vida en la vejez (Herrera, Barros y Fernández 2007). En ella se evaluaron la satisfacción de las personas mayores en relación a varios ámbitos.

Según este estudio quienes están más satisfechos son los que realizan actividades significativas como salir de la casa y tienen más habilidades psicosociales, pues les permiten ajustarse a las transformaciones propias del envejecer.

Otro punto que se señala también es que a mayor escolaridad hay una mejor calidad de vida, lo que se relaciona con un aumento de los ingresos, la posibilidad de un estilo de vida más saludable, recursos para salud, proporcionando a las personas habilidades psicosociales y cognitivas que tendrían un impacto positivo en las situaciones en donde se requiere cierto ajuste o adaptación.

Las mujeres son las menos satisfechas y las que tienen menor nivel de vida en varias dimensiones que fueron definidas como predictoras de calidad de vida: peor percepción de su salud, peor capacidad funcional básica, síntomas geriátricos, mayor frecuencia de dolores que interfieren en la vida diaria.

Otro estudio realizado en Chile (Mella, González, D'Appolonio, Maldonado, Fuenzalida y Díaz 2004), a diferencia del anterior, sostiene que las mujeres presentaban mayor bienestar subjetivo, siendo el género, el apoyo social percibido y la ausencia de depresión, los factores que determinan en un $54,3 \%$ dicho bienestar.

\section{Método}

\section{a) La perspectiva}

Desde la emergencia de los estudios sobre calidad de vida y su incorporación en las reflexiones disciplinarias, la sociedad ha incorporado nuevos valores y estilos de vida. En este marco, consideramos pertinente observar el concepto desde una perspectiva dinámica, que reconozca la complejidad de su conocimiento, definición y medición.

Nuestra reflexión busca articular el entendimiento de este concepto desde una perspectiva sociocultural amplia, que reconozca la importancia de las definiciones que comúnmente se han realizado, centrando nuestra mirada en un grupo etáreo particular en expansión: 
los adultos mayores. La selección de este grupo se basa en que tradicionalmente ha sido comprendido y tratado desde perspectivas vinculadas a la salud, siendo esto especialmente observable cuando se realizan estudios para medir su calidad de vida, en base a la presencia o ausencia de enfermedades, y deterioros físicos y mentales vinculados al proceso de envejecimiento.

\section{b) Las técnicas}

La estrategia metodológica se ha desarrollo contemplado el trabajo con dos técnicas de producción de datos cualitativos: grupos focales y entrevistas en profundidad. El grupo focal como técnica de producción de datos cualitativos, permite realizar una discusión grupal organizada alrededor de una temática específica ${ }^{3}$. Por su parte, la entrevista en profundidad consiste en una conversación guiada, la que permite alcanzar importantes niveles de intimidad y profundidad.

\section{c) La muestra}

Los estudios cualitativos trabajan con muestreos intencionales, es decir, que no siguen las leyes del azar. Esto significa que el investigador es quien selecciona las unidades de la muestra. La definición de nuestra muestra siguió las orientaciones del muestreo teórico una de las modalidades del muestreo intencional- , cuyo principio básico es generar teoría por medio de la colección, codificación y análisis de datos, en base a los cuales se va decidiendo los datos que se continuarán produciendo y en dónde encontrarlos. La idea, por lo tanto, ha sido profundizar en las categorías que se desea estudiar y producir la información más relevante para el concepto o teoría que se está elaborando: calidad de vida en personas mayores. El muestreo se da por finalizado cuando se alcanza el nivel de saturación, esto es, cuando dejan de aparecer nuevos conceptos y categorías relevantes. Las unidades o dimensiones elegidas son aquellas que garantizan mejor cantidad y calidad de la información. En definitiva, este tipo de muestreo permite que la muestra pueda ser alterada durante el proceso de producción de la información (Ruiz Olabuénaga 1999). El Cuadro № 1 presenta la estrategia de muestreo para ambas técnicas que se utilizaron.

Cuadro No 1. Criterios muestrales Grupos Focales y Entrevistas

\begin{tabular}{lll}
\hline \multicolumn{1}{c}{ Criterio } & \multicolumn{1}{c}{ Valores } \\
\hline a) Sexo & $>$ Masculino \\
& $>$ Femenino \\
\hline b) Edad & $>$ Tramo 1:60 a 75 años \\
& $>$ Tramo 2: 76 y más \\
\hline c) Nivel socioeconómico & $>$ Alto \\
& $>$ MSE) & $>$ Bajo \\
\hline d) Nivel de Autonomía & $>$ Autonomía-Autovalencia \\
& & $>$ Heteronomía-Semivalencia \\
\hline
\end{tabular}

\footnotetext{
${ }^{3}$ Se trata de un grupo artificial pues, aunque los integrantes puedan conocerse de antemano, el grupo conformado en torno a un tema seleccionado no existe ni antes ni después de la sesión de conversación (Álvarez-Gayou Jurgenson 2007)Es decir, aunque antes los integrantes del grupo hayan compartido instancias de conversación, es altamente improbable que lo hayan hecho en torno al problema que determina la conformación del grupo con fines investigativos, en nuestro caso, en torno a su calidad de vida
} 
Los grupos focales se realizaron a personas de 60 años y autovalentes. Por su parte, las entrevistas en profundidad se realizaron a personas de más de 60 años semiautovalentes, y tuvieron como propósito complementar la información obtenida en los grupos focales.

Los Grupos Focales se conformaron de la siguiente forma:

a) Grupos Focales Mixtos. Los integrantes fueron de ambos sexos, de diferente nivel socioeconómico y de ambos tramos etáreos. Se realizaron dos grupos mixtos iniciales, con el objetivo de realizar una discusión exploratoria, y un grupo mixto al finalizar la etapa de aplicación de esta técnica, con el propósito de discutir los resultados obtenidos durante esta fase de la investigación.

b) Grupos Focales con integrantes seleccionados, que se conformaron según la tipología indicada.

En total se realizaron 16 grupos focales, con un promedio de siete integrantes cada, y 10 entrevistas en profundidad de acuerdo a los criterios muestrales descritos.

El corpus de información producida fue analizado con el software para el análisis de datos cualitativos ATLAS- ti $5.0 \AA$

\section{Resultados}

\section{a) El concepto de calidad de vida según la perspectiva de las personas mayores}

Los resultados de la investigación permiten distinguir dos aspectos: la construcción del concepto de calidad de vida en las personas mayores, y las dimensiones asociadas al concepto. Si bien ambos aspectos se encuentran estrechamente interrelacionados, es necesario diferenciarlos, pues la definición que realizan las personas mayores se constituye en su perspectiva de comprensión de la calidad de vida, y será desde ahí donde realicen sus evaluaciones y generen sus expectativas sobre la calidad de de vida propia y la de la población adulta mayor.

Las definiciones entregadas varían en sus énfasis, pero de modo general el concepto puede resumirse como la constante vinculación, y muchas veces tensión, entre las condiciones individuales y las condiciones sociales. Podríamos decir que para las personas mayores la calidad de vida es una actitud positiva frente a la vida, la cual se ve determinada por las condiciones de salud y las relaciones interpersonales, pero también determinada por las condiciones estructurales presentes en la sociedad: derechos y oportunidades, y acceso a servicios básicos.

"Yo entiendo que calidad de vida es que cualquier persona adulto mayor, sea como sea, tiene que llegar a esta edad y poder tener atención de salud, locomoción, poder vivir y si no tiene casa poder pagar donde vivir, poder vestirse decentemente en general" (GF hombres, NSE medio, 60- 74 años).

"Yo considero calidad de vida tener buena salud; tener buena relación con las personas que uno tiene alrededor; tratar de pasarlo lo mejor posible, en lo posible y no pasar quejándose por enfermedades, quejarse cuando es muy necesario no más" (GF mujeres, NSE alto, 60- 75 años).

Al comenzar a identificar las diferentes dimensiones que se desprenden de esta definición, se observa la amplitud del concepto siendo casi omniabarcador. A modo de síntesis la Figura $\mathrm{N}^{\circ} 1$ muestra los ámbitos de la calidad de vida y sus dimensiones. 
Figura $\mathbf{N}^{\circ}$ 1. Dimensiones de la Calidad de Vida: definición según percepciones de las personas mayores.

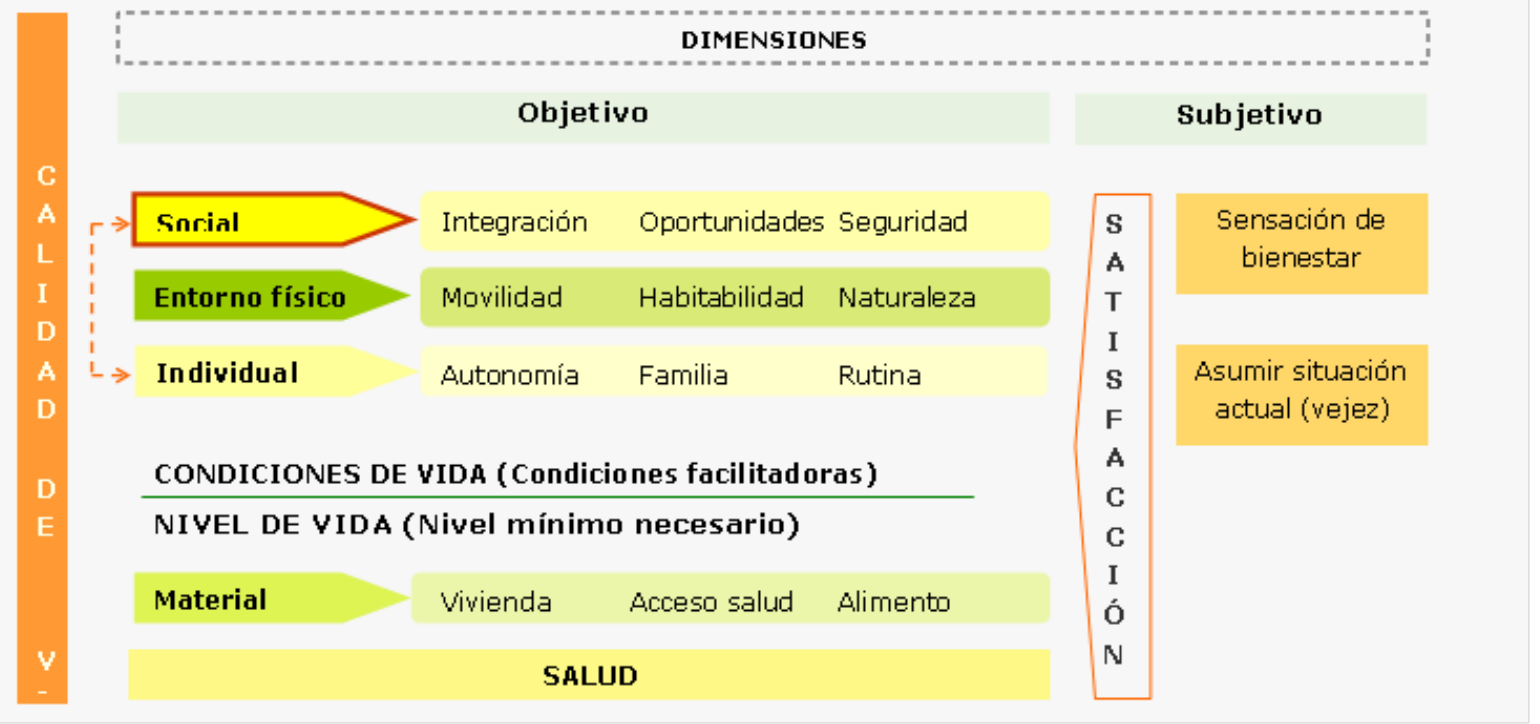

Del modo como es entendida la calidad de vida por las personas mayores se puede realizar una primera distinción identificando dos ámbitos: el objetivo y el subjetivo. Aunque se debe aclarar que el ámbito objetivo - constituido por la estructura de oportunidades- es también eminentemente subjetivo al momento de introducirse las evaluaciones sobre la incidencia que las dimensiones de este nivel tienen en la calidad de vida de cada persona mayor.

En tal sentido, las propias personas mayores reconocen el carácter subjetivo del concepto, que hace difícil el establecimiento de parámetros, y por lo mismo se vuelve interesante para reflexionar sobre las formas de vida de otras personas mayores y los parámetros propios para su evaluación.

"...hay gente que no tiene ni una cosa, apenas para tomarse una taza de té y ipucha que los ves felices! Eso es algo tan personal" (GF mujeres, NSE medio, 60- 74 años).

Lo anterior no debe entenderse como ingenuidad y conformismo, pues siempre se reconoce que la estructura de oportunidades de cada persona puede facilitar la percepción de una buena calidad de vida.

"La parte económica también es muy importante, porque sin dinero, aunque sea poco, no hay salud, ni amistad y tampoco se ven las cosas muy positivas cuando no hay dinero. Son todas cosas que se unen unas con otras. Y si uno está contento la familia también está contenta, y si uno es el pilar de la familia, tiene hijos, nietos, se lleva una casa alegre, positiva todos vana a estar en ese mismo ambiente" (GF mixto, 60- 74 años).

El nivel objetivo está constituido en primer lugar por las necesidades básicas que deben ser satisfechas, las cuales se han definido como el nivel de vida ${ }^{4}$, el cual está conformado en primer lugar por la dimensión de salud - entendida como la posibilidad de mantener la autonomía- , y por la dimensión material que tiene que ver con el acceso a vivienda, salud

\footnotetext{
${ }^{4}$ De acuerdo a una revisión bibliográfica realizada por Paula Aranibar (2001) "se refiere sólo a aspectos económicos y materiales en que se desenvuelve la vida: incluye salario, propiedades, equipamiento, vivienda, en fin, la capacidad de consumo en su sentido más amplio".
} 
y alimentación adecuada a las necesidades de la edad. Pese a la subjetividad del concepto, existe cierto consenso entre los participantes, de que antes de hablar de calidad de vida se deben tener estas dimensiones solucionadas o al menos bajo un nivel aceptable de control.

Se pueden distinguir en segundo lugar en el nivel objetivo, lo que se ha denominado condiciones de vida (contexto y situación personal observable), las cuales están constituidas por las dimensiones: social, entorno físico e individual, las cuales a su vez permiten distinguir subdimensiones. Es por ello que las condiciones de vida pueden definirse como condiciones facilitadoras para tener una buena calidad de vida, sin que todas ellas deban ser total o parcialmente cubiertas para que una persona pueda generar una evaluación positiva de su situación individual. A modo de ejemplo, una persona puede indicar que tiene una muy buena calidad de vida, y que eso se debe a que es autónoma, cuenta con buenas relaciones familiares y no tiene problemas para movilizarse por la ciudad para realizar su rutina diaria, sin embargo para esta persona no resulta relevante el contar en esta etapa con oportunidades para integrarse a la sociedad (tales como el trabajo).

El nivel subjetivo podría ser entendido como la evaluación constante de las dimensiones anteriores, en base a experiencias propias de la biografía individual y la influencia del medio sociocultural, ambos inciden en los parámetros utilizados por cada persona mayor para evaluar su calidad de vida propia y la de las personas mayores del país. Es por ello que se define principalmente como la satisfacción con respecto a una o varias de las dimensiones objetivas constituyentes de la calidad de vida. Esta sensación de satisfacción está conformada por la sensación de bienestar, que corresponde a una disposición positiva frente a la vida y tranquilidad espiritual; y por generar un parámetro inicial desde donde medir la calidad de vida de acuerdo a la situación personal influida directamente por la etapa de la vida en la cual estas personas se reconocen: la vejez. Así, el segundo elemento que configura la satisfacción es el asumir esta situación actual para generar indicadores propios para evaluar la calidad de vida.

\section{b) Dimensiones de la calidad de vida}

Como indica la Figura $\mathrm{N}^{\circ} 1$ se identificaron cinco dimensiones principales: salud, material, individual, entorno físico y entorno social. La primera dimensión tiene que ver con la presencia de enfermedades físicas y mentales que puedan repercutir en el nivel de autovalencia de las personas mayores. La dimensión material, incluye los bienes y el acceso a servicios; mientras que la dimensión individual tiene que ver con el mundo privado de las personas mayores, y por tanto corresponde a aquellos aspectos que pueden ser gestionados individualmente. En tal sentido, la dimensión individual incluye la autonomía, entendida como independencia económica y emocional con respecto a terceros para mantener un estilo de vida de acuerdo a los intereses propios. Se incluye también en la dimensión individual las relaciones con la familia y la posibilidad de mantener o crear una rutina diaria propia, en este sentido, esta última subdimensión de vincula estrechamente con la autonomía.

La dimensión entorno físico corresponde principalmente a las condiciones de infraestructura de la ciudad donde se habita, de las posibilidades que ésta entrega a las personas mayores para poder desplazarse en cualquier medio de transporte. Asimismo, incluye el reconocimiento de la importancia de contar con áreas verdes y espacios públicos que permitan realizar actividades recreativas, que sean a su vez accesibles para las personas mayores. El entorno físico, en un nivel más micro, incluye también las condiciones de las viviendas y la adecuación de sus características para las necesidades particulares de las personas mayores que las habitan. 
Por último, la dimensión social incluye las oportunidades presentes en la sociedad chilena que facilitan o inhiben la integración de las personas mayores. Es por ello que esta dimensión incluye no sólo la posibilidad de participar en centros y clubes, sino también las condiciones para participar en instancias valoradas por la sociedad-como es el caso del trabajo- , o para la participación a un nivel más político donde las personas mayores pedan influir en la toma de decisiones. Siendo uno de los hallazgos más significativos de la investigación el haber identificado la importancia que esta dimensión tiene sobre la calidad de vida de las personas, a continuación profundizaremos en los diferentes ámbitos que la conforman.

\section{c) Dimensión Social: Calidad de Vida y Entorno Social}

Podemos ampliar lo que se ha definido como entorno social, comprendiéndolo como las relaciones establecidas entre las personas mayores con su entorno próximo (familia, amigos, vecinos) y extenso (barrio, municipalidad, ciudad, estereotipos, etc.) que posibilitan o dificultan su calidad de vida y sensación de bienestar. Desde la perspectiva del capital social, podemos afirmar que tener buenas redes familiares y de amistades y conocidos, es esencial para la calidad de vida, en tanto estas redes permiten aumentar las capacidades de las personas para manejar sus vidas (Bezanson, 2006)

La dimensión social se vincula estrechamente con lo que se describió anteriormente como dimensión individual a través del reconocimiento de la importancia de la calidad de los lazos familiares en la calidad de vida propia. La familia, así como los amigos y conocidos, son parte de las redes de las personas mayores, donde la calidad de las relaciones pude afectar positiva o negativamente su calidad de vida (Herrera y Kornfeld, 2008).

"Lo mismo, que este bien el grupo familiar le hace feliz a uno y eso le permite disfrutar de una salida, ir a tomarse un café con una amiga" (GF mixto, 60- 74 años).

En tal sentido, las dimensiones identificadas son coincidentes con otras investigaciones como el realzado por Cardona y otros (2002) en Medellín Colombia cuyos resultados muestran que la calidad de vida del adulto mayor de la ciudad es explicada en un $97 \%$ por los componentes: autonomía, seguridad social-economía y redes de apoyo social. Apoyando lo anterior, los resultados de grupos focales desarrollados en Canadá basados en la pregunta"¿qué cosas afectan sus vidas para bien o para mal?" (Bryant y otros. 2002: 71), indicaron que los contactos y las redes sociales son parte de los principales factores reconocidos por las personas mayores. Asimismo, menos nombrados, pero igualmente relevantes se indicó el viejismo presente en la sociedad y la falta de voluntad política frente a temáticas relevantes vinculadas a la vejez.

\section{d) Familia}

Se reconoce como uno de los aspectos centrales a la hora de evaluar la calidad de vida propia, siendo que las relaciones familiares se vinculan de diferente manera a la evaluación de la calidad de vida.

"Que la familia este bien, ya a la edad de uno eso es lo que uno piensa siempre y hacer las cosas que a nosotros nos gusta" (GF mixto, 60- 74 años).

Uno de los puntos más interesantes en relación al papel que desempeña la familia en la evaluación de la calidad de vida de las personas, es que la calidad de vida de familiares, sobre todo de hijos y nietos, es incluida en la evaluación de la propia calidad de vida. En tal sentido el bienestar subjetivo y a la vez la satisfacción que los adultos mayores pudieran sentir al relacionar su situación actual personal y logros en relación a sus 
expectativas se ve afectado en gran medida por la situación y los logros de los familiares más cercanos, lo que tiene como resultado que el bienestar pueda teñirse con tintes amargos o dulces, según como se encuentre la familia.

"ella vive pendiente de los niños. A qué hora descansa, no descansa por que estamos todos acostados y ella todavía dándose vueltas, viendo una y otra cosa. Esas son las cosas que me hacen sufrir" " (M, NSE Medio, 86).

De manera transversal también aparece la dependencia en las relaciones familiares como un elemento performativo de éstas. Asiduamente nos encontramos con dos tipos de situaciones, aquellas en donde el o la adulta mayor depende en distinto grado de un familiar, sea éste hijo, nieto, pareja, entre otros; o aquella en donde uno o varios familiares dependen en grados variables del o la adulta mayor.

Cuando es el adulto mayor el que depende de los familiares, su bienestar va a estar supeditado en gran medida al tipo de relación que mantenga con dichas personas. Son éstas personas las que aseguran las condiciones mínimas para la vida en términos de alimentación, movilidad, aseo, entre otras cosas. A la vez se transforman en los pilares afectivos, brindando compañía y afecto o siendo sindicados como los culpables de la falta de ambas cosas.

"Quien va a vivir con lo que estamos viviendo, porque yo misma imagínese con esa pensión que saco, no me alcanzaría nada si no es por mi hija que me ayuda" (M, NSE Bajo, 67).

Cuando la relación de dependencia es al revés, es decir, cuando son los hijos y nietos los que dependen de los adultos mayores aparecen dos situaciones que afectan de manera diferencial la calidad de vida. Si la dependencia, que en la mayoría de los casos es económica, es vivida como producto de una decisión propia, aparece como fuente de satisfacción. Si por el contrario, la dependencia resulta según la propia percepción como no voluntaria y sin posibilidad de decidir al respecto, es vivida como una suerte de abuso, bajo la idea de haber cumplido con una "tarea" de crianza de hijos y nietos, siendo ellos quienes deben enfrentar el futuro, y por tanto proyectar una labor comenzada inicialmente por las personas mayores.

"Yo pasé el aviso y ahora nadie me molesta, salvo contadas ocasiones en que falla la nana, de vez en cuando. Yo dije a los 70 años yo me jubilo, no cuido mas nietos, y el menor de mis nietos que tiene 17 años me dijo "nona, y quien me va a criar mis hijos". Yo creí nietos y bisnietos". (Mujer, NSE medio, 60-74)

Existen opiniones divididas sobre si los hijos deben encargarse de sus padres cuando ya comiencen a necesitar más cuidados especiales producto de problemas de salud, algunas personas mayores prefieren ser atendidos en casas de reposo especializadas para mantener cierta autonomía, y ser visitado por sus hijos, otros mencionan esta opción para no ser una carga para su familia. Asimismo, otro grupo importante de personas mayores indica como central el que sea su familia, hijos principalmente, quienes se encarguen de cuidarlos, sintiendo que el delegar esta responsabilidad a una institución es ser abandonados.

"Y resulta que como calidad de vida para mi yo he buscado un hogar, lo tengo hasta listo, el Hogar de los italianos" (GF mujeres, NSE medio, 60- 74 años).

"Por muy bueno que sea el hijo, la nuera o lo que sea, no la puede tener en la otra casa no porque no la pueda tener, la puede tener pero uno tiene que respetar el orden y la forma de vida que tienen en su casa" (GF, mujeres, NSE alto, 75- >75 años). 
Un tema que se torna relevante, si lo consideramos desde una perspectiva temporal, el papel que juega la viudez en la calidad de vida de las personas. La muerte de la pareja constituye un hito, un quiebre que tiene diferentes consecuencias según cada caso. Es curioso constatar que en las mujeres la viudez puede ser vivida como la apertura a un mundo al que no habían tenido acceso antes, marcado por la autonomía, la que es valorada positivamente. En el caso de los hombres se observa un fenómeno muy diferente. Muchos de ellos viven la viudez como una pérdida que los arroja a la soledad y a la carencia. Esta vivencia diferenciada de la pérdida de la pareja debe ser inscrita y pensada en el contexto de la separación de roles de género tradicional, en la que la mujer era relegada al espacio privado y se ocupaba de las labores de la casa y el hombre habitaba mayoritariamente el espacio público y hacía las veces de proveedor. El quedarse solos conlleva pérdidas y ganancias, lo que parecen distribuirse de forma diferente según el rol de género que se haya cumplido durante la vida.

"quede solo hace 3 años y me la he visto bien afligido solo, y si no sabe cocinar, no sabe hacer nada peor todavía. Así que de a poco aprendí a hacerme la comida, lavar mi ropa, planchar a veces, a veces no, asear la casa, mantenerse alerta y por ahí saco unos pollitos, unos trabajos por ahi para entretenerme y no estar tan solo".(Hombre, NSE Medio, 60-74)

\section{e) Relaciones entre pares}

Se reconoce la importancia de contar con amigos y amigas, los cuales muchas veces pueden prestar más apoyo que la misma familia.

"Para mi es tener buena salud compatible con el medio y tener un grupo importante de amigos con mucho cariño. La familia viene por añadidura, las amigas las escoge uno" (GF mixto, 60- 74 años).

El reunirse con su grupo de pares, ya sea que pertenezcan o no a algún grupo de adultos mayores, les ayuda a mantener una rutina de recreación y compañía, compartiendo momentos de la cotidianeidad que les permiten conversar sobre asuntos personales y compartir. El compartir vivencias comunes y una etapa de la vida común, hace que el mantener o crear redes en la vejez sea muy valorado.

"Yo tengo amigas de 40 y 50, y todavía somos amigas. He hecho amistad acá (...) hace 15 días y tuve el apoyo de mis compañeros del circulo, y mis compañeras, cuando falleció mi hermano. $Y$ con ellas nos juntamos y vamos al cine, vamos a tomar tecito, su cafecito y nos juntamos" (GF mixto, NSE alto, 75- > 75 años).

En la definición de calidad de vida muchas personas mayores aluden a evitar aislarse, generar estrategias que les permitan vincularse con la sociedad. En este marco, el poder mantener o crear lazos de amistad es valorado en tanto permite evitar la soledad.

"La persona que se aísla; yo conozco gente sin amigos o familiares es una persona que esta amargada, explotan por cualquier cosa, todo es negativo, no tiene otro mundo que sus 4 paredes. El señor que vende pan en la esquina, la verdulera y punto" (GF mixto, 60- 74 años).

"Yo creo que (...), hay personas que se aíslan, se quedan en la casa, no tratan de hacer amistades, son gente que... ya estoy vieja, para que" (GF mixto, NSE alto, 75- > 75 años). 


\section{f) Relaciones con generaciones más jóvenes}

La mayoría de los entrevistados y entrevistadas indican una relación compleja con los jóvenes que tendría varias aristas, vinculadas mayoritariamente a la desconfianza hacía las generaciones más jóvenes y a la sensación de desvalorización que dicen experimentar.

"Y el primer insulto que sale de la gente joven es vieja o viejo tal por cual; claro que lo pasaste a llevar o que se yo... viejo, el insulto mas grande que tienen. A la vez el trato con ellos, siempre que sea respetuoso, genera mucha satisfacción, pues se considera que ambos pueden aprender y salir beneficiados de dicha relación" (GF mixto, 60- 74 años).

A la vez el trato con ellos, siempre que sea respetuoso, genera mucha satisfacción, pues se considera que ambos pueden aprender y salir beneficiados de dicha relación.

"Trabajo con clientes que me llevo bien con ellos, me siento bien porque saliendo de la casa, y son gente joven, no tengo problemas, me echan tallas, yo también la echo, me cuentan chistes, la hacen reír a uno" (GF mixto, 60- 74 años).

En relación a la desconfianza, las personas mayores suelen reprobar el estilo de vida que actualmente llevan los más jóvenes. Se refieren con frecuencia a la pérdida de valores, a la drogadicción a la delincuencia como los grandes males que los aquejan.

"Parece que la juventud está demasiado cómoda, lo único que quieren es pasarlo bien a costa de quien sea" (H, NSE Alto, 80).

De forma recurrente se sienten desvalorizados y reportan que no sienten que exista una relación de respeto o el trato se basa en una serie de prejuicios negativos que dificultan el establecer una relación.

"Como que ellos nunca van a llegar a la edad que uno tiene, eso lo encuentro como una falta de respeto. A mi me toco escuchar una vez a una persona joven, y habían bastantes personas mayores, y dijo: a mi me cargan los viejos. Entonces es duro, y es una persona educada" (GF mixto, 60- 74 años).

Pese a lo anterior, se reconoce la importancia de relacionarse con otras generaciones, pues se siente una responsabilidad en la trasmisión de experiencia, y una posibilidad de que los jóvenes les enseñen cosas nuevas que les permitan mantenerse vinculados con la sociedad.

"Si te fijas que en estos momentos como adultos mayores estamos fuera de un montón de cosas tecnológicas, que en estos momentos están siendo un idioma como la internet, la computación, algunos otros adelantos que hay de distinto tipo, tanto medico... que vas quedando fuera si no te relacionas con gente joven que te vaya enseñando todo esto, $y$ además ir entregándole la experiencia no solo a nuestros nietos sino que pudiéramos entregar a los niños, porque son los niños que más tarde $o$ en otras generaciones que vengan después de nosotros van a poder ser mas consientes de nuestras necesidades" (GF mujeres, NSE medio, 60- 74 años).

\section{g) Sociedad y cotidianeidad}

Las personas mayores indican que existiría una percepción negativa de ellos basada en estereotipos, lo que se traduce en un trato también negativo y muchas veces en situaciones donde intentar engañarlos 0 aprovecharse de ellos. Asimismo, los 
entrevistados y entrevistadas perciben un trato de discriminación en diversos aspectos de su vida diaria, en actividades que realizan y en opiniones.

"vas a comprara cualquier cosa y te tratan de engañar, de darte menos vuelto, ¿se han fijado?, al comprar un boleto de metro tiras un billete de mil y tratan de darte menos plata, un cajero de metro. ¿A cuántos adultos nos engañan al día?" (GF hombres, NSE bajo, 60- 74 años).

Al respecto, y en estrecha relación con el entorno físico, pueden mejorar su calidad de vida las decisiones que se tomen a nivel de municipalidades, siendo muy diferente la calidad de vida de cada persona según la comuna donde resida, pues dependen de la importancia que les den como grupo o que decidan en torno a problemáticas que para ellos son esenciales, a la hora de tomar las decisiones. Sin embargo también se reconoce la importancia de las propias personas mayores para producir cambios a su favor, debiendo insertarse más en su entorno social, en los espacios donde se toman las decisiones.

"Vivimos en una comuna privilegiada. En Ñuñoa tenemos polideportivo, varios parques, Grecia, Juan 23, hay varios parques alrededor" (GF mixto, 60- 74 años).

El entorno social ligado al barrio también es un aporte a mejorar su calidad de vida, pues independiente que sean amigos o no, se precia a los vecinos los cuales brindan seguridad y apoyo.

"Es bien importante, por ejemplo yo vivo en un barrio residencial donde tengo muy buenos vecinos. Estoy tranquila porque sé que mi vecino de al lado está pendiente de mi casa y la siente igual y todos somos vecinos muy unidos, un barrio bien tranquilo" (GF mixto, 60- 74 años).

\section{Conclusiones}

Dentro de la multiciplicidad de factores que los adultos mayores identificaron como componentes de la calidad de vida en la vejez, se ha podido observar que el entorno social aparece uno de los más influyentes. Esto porque puede limitar la participación efectiva en espacios de decisión y generar situaciones de viejismo, como también, puede articular y concentrar recursos tales como la contención emocional, apoyo, recursos económicos, materiales, incentivar a la actividad, articular la rutina diaria, facilitar la actualización de cambios sociales y relaciones generacionales, y ayudar al desenvolvimiento de los adultos mayores en el entorno físico en el que viven.

Resulta particularmente relevante la comprensión del concepto calidad de vida construido a partir de las reflexiones de las personas mayores, pues agrega a los aspectos y dimensiones generalmente referidos por la literatura especializada, la evaluación subjetiva de éstos, la cual se basa en el reconocimiento de su condición de persona mayor, entregando matices novedosos para la comprensión de los factores identificados.

Los resultados de la investigación dan cuenta de la complejidad del concepto de calidad de vida, sobre todo al observarlo en este grupo etáreo en particular, puesto que se conjugan la trayectoria vital y el momento biográfico actual de las personas. Lo anterior nos ha llevado a una aproximación del concepto entendido como calidades de vida. Y ello no sólo por su multidimensionalidad y por la conjunción objetivo subjetivo, sino más bien, por lo heterogénea y dinámica que resulta la vejez como realidad histórico social y biográfica individual (Osorio 2006). RM 


\section{Bibliografía}

Aranibar, P. (2004). Calidad de Vida y Vejez. Universidad de Barcelona. Extraído el 26 de junio de 2009 desde www.ciape.org.br/ .../ CALIDADDEVIDAYVEJEZ\%B1\%D.doc

Bezanson, K. (2006). Gender and the limits of social capital. Canadian Review of Sociology and Anthropology, 43(4), 427-443.

Bryant, T., Rápale, D., Brown, I., Cogan, T. Dallaire, C. Laforest, S. et. Als. (2002). A Nation for all ages?. A partocipatory study of Canadian Seniors' Quality of Life in Seven Municipalities. York University, Centre for Health Studies. School of Health Policy and Management. Toronto, Canada: School of Health Policy and Management.

Cardona, D., Estrada, A. \& Agudelo, H. (2002). Envejecer nos "toca" a todos: Caracterización de algunos componentes de calidad de vida y de condiciones de salud de la población adulta mayor de Medellín. Medellin, Colombia: Editorial Lvieco e hijas

CELADE. (2006). División de Población de la CEPAL. América Latina y el Caribe: Desafíos y oportunidades de una sociedad que envejece. Temas de Población y Desarrollo, (5). Extraído el 20 de abril de 2009 desde http:// www.eclac.cl/ celade/ noticias/ noticias/ 7/23867/ PyDEN_5.pdf

Dijkers, M. (2007). "What's in a name" The indiscriminate use of the "Quality of life" label, and the need to bring about clarity in conceptualizations. International Journal of Nursing Studies. 44(1), 153- 155

Hernández P., A. (2004). Las personas con discapacidad. Su calidad de vida y la de su entorno. Revista Aquichan, 4(4), 60- 65.

Herrera, M. y Kornfeld R. (2008). Relaciones familiares y bienestar de los adultos mayores en Chile. Revista En Foco - Expansiva, (131), 1- 15.

Herrera, S. Barros, C. y Fernández, B. (2007). Primera Encuesta Nacional de Calidad de Vida en la Vejez. Principales resultados. Instituto de Sociología e Instituto de Economía. Santiago de Chile: Pontificia Universidad Católica de Chile.

Hidalgo-Rasmussen, C. (2008). De los Comportamientos de riesgo a la Calidad de vida de los Adolescentes. Guadalajara: Editorial Universidad de Guadalajara.

Higgs, P., Hyde, M., Wiggins, R., Blane, D. (2003). Researhing QoL in early old age: the importance of the sociological dimension. Social Policy \& Administration, 37(3), 239252.

Mella, R.; González, L.; D'Appolonio, J; Maldonado, I.; Fuenzalida, A; \& Díaz, A. (2004). Factores Asociados al Bienestar Subjetivo en el Adulto Mayor. Psykhe, 13(1), 79- 89.

Moreno, Julia. (2004). Mayores y calidad de vida. Portularia, 4(1), 187- 198.

Moons, Philip. Budts, Werner y De Geest, Sabina. (2006). Critique on the conceptualization of quality of life: A review and evaluation of different conceptual approaches. International Journal of Nursing Studying, 43(7), 891- 901.

Osorio, Paulina. (2006). La longevidad: más allá de la biología. Papeles del CEIC - Centro de Estudios sobre la Identidad Colectiva, Universidad del País Vasco, (22). Extraído el 9 de Julio de 2009 desde http:// www.identidadcolectiva.es/ pdf/22.pdf 
Pedrero, E. (2001). La calidad de vida y las personas mayores. Monografía presentada a los seminarios de psicogerontología.

Extraído el 2 de Julio desde http:// psiconet.com/tiempo/monografias/ calidad.htm

Puts, Shekary, Widdershoven, Heldens, Lips y Deeg. (2007). What does quality of life mean to older frail and non- frail community-dwelling adults in the Netherlands?. Quality of Life Research, 16(2), 263-277.

Sanduvette, Sandra. (1990). Calidad de vida en los adultos mayores. Anales de psicología, $6(2), 147-158$

Schwartzmann, L. (2003). Calidad de Vida relacionada con la Salud: Aspectos conceptuales. Ciencia y enfermería, 9(2), 09- 21.

Walker, A. (2005). Understanding Quality of Life in Old Age. Maidenhead: Open University Press.

\section{Sobre las autoras}

Paulina Osorio Parraguez es Doctora en Sociología. Profesora e investigadora del Departamento de Antropología de la Facultad de Ciencias Sociales de la Universidad de Chile. Entre sus últimas publicaciones se encuentran: (Junto a M. J. Torrejón, N. Vogel y M. S. Anigstein) Ageing and social environment: latent discriminations. The case of Chile (The Journal of Nutrition, Health \& Aging, 2009) y Mujeres de la tercera edad y su relación con el trabajo: expectativas de calidad de vida (Brasil, 2009)

posorio@uchile.cl

María José Torrejón es Magíster en Antropología y desarrollo. Investigadora Programa Domeyko Envejecimiento, Universidad de Chile, Facultad de Ciencias Sociales de la Universidad de Chile. Entre sus últimas publicaciones se encuentran: (Junto a P. Osorio, N. Vogel y M. S. Anigstein) Ageing and social environment: latent discriminations. The case of Chile (The Journal of Nutrition, Health \& Aging, 2009) y (Junto a I. Espinoza, P. Osorio, N. Vogel y M. S. Anigstein) Perception of health factors affecting the quality of life in elderly people in Santiago, Chile (The Journal of Nutrition, Health \& Aging, 2009) matorrej@uchile.cl

María Sol Anigstein es Magíster en Antropología y Desarrollo. Investigadora Programa Domeyko Envejecimiento, Universidad de Chile, Facultad de Ciencias Sociales de la Universidad de Chile. Entre sus últimas publicaciones se encuentran: (Junto a P. Osorio, N. Vogel y M. J. Torrejón) Ageing and social environment: latent discriminations. The case of Chile (The Journal of Nutrition, Health \& Aging, 2009) y (Junto a I. Espinoza, P. Osorio, N. Vogel y M. J. Torrejón) Perception of health factors affecting the quality of life in elderly people in Santiago, Chile (The Journal of Nutrition, Health \& Aging, 2009)

msanigste@uchile.cl

\section{Contacto}

Facultad de Ciencias Sociales

Universidad de Chile

Cap. Ignacio Carrera Pinto 1045.

Ñuñoa, Santiago de Chile

Recibido: Marzo 2011

Aceptado: Mayo 2011

Artículo disponible en: http:// www.facso.uchile.cl/publicaciones/mad/24/ osorio03.pdf 